Disponível em

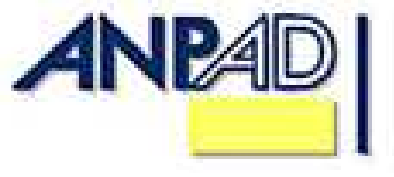

http://www.anpad.org.br/rac

RAC, Rio de Janeiro, v. 17, n. 2, art. 6, pp. 239-262, Mar./Abr. 2013

$(\mathrm{cc}) \mathrm{EY}-\mathrm{NC}$

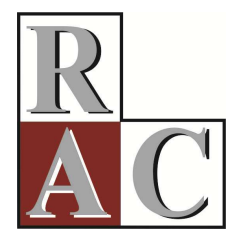

\title{
Efeitos da Internacionalização sobre o Desempenho de Multinacionais de Economias em Desenvolvimento
}

\section{Effects of Internationalization on the Performance of Multinational Companies from Developing Economies}

\begin{abstract}
Alisson Maxwell Ferreira de Andrade E-mail: alissona@fearp.usp.br Faculdade de Economia, Administração e Contabilidade de Ribeirão Preto - USP Av. Bandeirantes, 3900, Monte Alegre, 14040-900, Ribeirão Preto/SP, Brasil.

Simone Vasconcelos Ribeiro Galina E-mail: svgalina@usp.br Faculdade de Economia, Administração e Contabilidade de Ribeirão Preto - USP Av. Bandeirantes, 3900, Monte Alegre, 14040-900, Ribeirão Preto/SP, Brasil.
\end{abstract}




\title{
Resumo
}

A relação entre o grau de internacionalização e o desempenho das empresas multinacionais (EMNs) tem sido amplamente estudada na literatura de negócios internacionais. Entretanto, a maior parte dos estudos é realizada com empresas dos países desenvolvidos. De modo a contribuir com o desenvolvimento da teoria de internacionalização-desempenho, este artigo mostra uma análise dessa relação no universo das EMNs de economias em desenvolvimento. O principal objetivo do trabalho é avaliar se a estratégia de expansão internacional dessas EMNs está sendo traduzida em forma de eficiência e eficácia na gestão do negócio da empresa. A amostra do estudo corresponde a 33 das 100 empresas multinacionais (EMNs) mais internacionalizadas de economias em desenvolvimento, sendo que as informações levantadas referem-se a um período de 6 anos (2004-2009). Os dados foram obtidos junto a duas fontes: (a) United Nations Conference on Trade and Development (UNCTAD); e (b) Thomson Reuters Datastream. Após análises de regressão múltipla, método dos mínimos quadrados, bem como análise de dados em painel, verificou-se uma relação linear-negativa entre o grau de internacionalização e o desempenho das EMNs de economias em desenvolvimento, indicando que quanto maior o grau de internacionalização, menor tende a ser o desempenho dessas empresas.

Palavras-chave: internacionalização de empresas; grau de internacionalização; desempenho.

\begin{abstract}
The relationship between the degree of internationalization and performance of multinational corporations (MNCs) has been widely studied in international business literature. However, most studies have been performed with companies from developed countries. In order to contribute to the development of the theory of internationalization-performance, this study examines this relationship in the universe of MNCs from developing economies. The main objective of this study is to analyze if these MNCs' international expansion strategies are being translated in an efficient and effective way in terms of managing company business. Our sample includes 33 of the 100 most highly internationalized multinational companies (MNCs) from developing economies, and the information was collected over a 6 year period (2004-2009). Data were obtained from two sources: (a) the United Nations Conference on Trade and Development (UNCTAD); and (b) Thomson Reuters Datastream. Multiple regression analysis, Ordinary Least Squares (OLS) method, and panel data analysis found a negativelinear relationship between the degree of internationalization and developing economy MNC performance, indicating that the higher the degree of internationalization, the lower MNC performance tends to be.
\end{abstract}

Key words: firm internationalization; degree of internationalization; performance. 


\section{Introdução}

Desde 1970, verifica-se uma tendência crescente de investimento direto estrangeiro (IDE) realizado pelas Empresas Multinacionais (EMNs) de economias em desenvolvimento. O montante de IDE realizado por essas EMNs saltou de US\$ $51 \mathrm{mi}$, em 1970, para US\$ 388,1 bi, em 2010 (United Nations Conference on Trade and Development [UNCTAD], 2011). Ainda de acordo com o mesmo relatório da United Nations Conference on Trade and Development (UNCTAD), essas EMNs aderiram à competição global em suas estratégias, já que, em 2010, seis economias em desenvolvimento estiveram entre as vinte que mais fizeram IDE no mundo.

Para Dunning (2000), uma das razões que leva as empresas a investirem no exterior é a busca por eficiência. Portanto, é importante avaliar se a expansão internacional exerce influência sobre o desempenho das EMNs de economias em desenvolvimento, foco deste artigo. Desde a fundação da área de negócios internacionais como campo de pesquisa, a maior parte dos estudos baseia-se na suposição de que a estratégia de expansão internacional é boa para o desempenho de uma empresa (Contractor, Kundu, \& Hsu, 2003). O que tem sustentado essa premissa são os significativos benefícios que a internacionalização pode conferir a uma empresa, tais como: economias de escala, acesso à mão de obra barata e redução de custos (Grant, 1987; Kogut, 1985). Embora existam muitas considerações que levem a previsões de um relacionamento positivo entre o grau de internacionalização e o desempenho, diversos outros fatores levam a acreditar que possa haver também um impacto negativo do grau de internacionalização sobre a rentabilidade da empresa. Por exemplo, Geringer, Beamish e Dacosta (1989) argumentam que a internacionalização pode implicar em custos associados a questões como coordenação e controle, bem como sistemas administrativos para a gestão de mercados culturalmente distintos.

Nesse sentido, vários têm sido os estudos realizados acerca dos efeitos da expansão internacional sobre o desempenho da empresa, entretanto, a maior parte deles tem sido realizada com EMNs de economias desenvolvidas, e os seus resultados não têm sido convergentes. Há estudos que apresentam uma relação linear positiva (Grant, 1987; Han, Lee, \& Suk, 1998; S. W. Kim \& Lyn, 1987); outros uma relação linear negativa (Brewer, 1981; Collins, 1990); ainda alguns que encontram relação em forma de $\mathbf{U}$ (Ruigrok \& Wagner, 2003); também relação em forma de $\mathbf{U}$ invertido (Daniels \& Bracker, 1989; Geringer, Beamish, \& Dacosta, 1989; Gomes \& Ramaswamy, 1999); e a literatura apresenta, ainda, relação curvilinear em forma de $\mathbf{S}$ horizontal, denominada teoria dos três estágios de internacionalização (Contractor et al., 2003).

Também apresentando resultados que não convergem, são poucas as pesquisas sobre a relação internacionalização-desempenho no universo das EMNs de economias em desenvolvimento em comparação aos estudos realizados com EMNs de economias desenvolvidas (Chang, 2007; Chiao \& Yang, 2011; Gaur \& Kumar, 2009; H. Chen \& HSU, 2010; Lin, Liu, \& Cheng, 2011; S. Chen \& Tan, 2012; Thomas, 2006). Além disso, essas pesquisas, em geral, focam um país ou uma região, portanto, sem considerar EMNs com diferentes países de origem.

Considerando o panorama apresentado, o intuito deste artigo é o de contribuir com o avanço na literatura na área, a partir da incorporação da análise de EMNs de economias em desenvolvimento, já que alguns autores afirmam que elas se comportam de maneira distinta das EMNc tradicionais no processo de internacionalização (Luo \& Tung, 2007; Mathews, 2006).

Dessa forma, o presente trabalho tem por objetivo testar a relação internacionalizaçãodesempenho nas mais internacionalizadas EMNs de economias em desenvolvimento, a fim de responder à questão: quais os efeitos do grau de internacionalização sobre o desempenho das empresas multinacionais de economias em desenvolvimento? 


\section{Fundamentação Teórica}

\section{Os benefícios da internacionalização}

A literatura de negócios internacionais tem apresentado diversos benefícios inerentes à estratégia de expansão internacional das empresas. Kobrin (1991) argumenta que, ao operar internacionalmente, a empresa pode se beneficiar de maior padronização de produtos, racionalização da produção e alocação mais eficiente dos recursos. Isso é possível porque uma empresa, ao operar além de suas fronteiras nacionais, tem a oportunidade de explorar os benefícios advindos da internalização nos mercados internacionais (Buckley \& Casson, 2009; Rugman, 1986), como economias de escala e escopo (Grant, 1987; Grant, Jammine, \& Thomas, 1988; Kogut, 1985). O paradigma eclético proposto por Dunning (1988) tem essa como uma de suas teorias estruturantes.

Adicionalmente, Grant (1987) argumenta que os seguintes benefícios podem levar o grau de internacionalização de uma empresa a ser positivamente relacionado com o seu desempenho: (a) retorno sobre os ativos intangíveis; (b) poder de mercado conferido pela atuação em âmbito internacional; (c) capacidade de realizar investimentos de risco; e (d) ampliação das oportunidades de investimento. Há ainda a possibilidade de prospecção de novos mercados, o que permite maior crescimento (Buhner, 1987; Han et al., 1998), além dos resultantes benefícios relacionados ao poder de barganha (Grant, 1987; Sundaram \& Black, 1992). Por outro lado, ao atuarem além de suas fronteiras nacionais, as empresas também podem se beneficiar da aprendizagem, do conhecimento e do desenvolvimento de capacidades ao redor do mundo (Kogut, 1985; W. C. Kim, Hwang, \& Burgers, 1993; Wernerfelt, 1984).

Levando em consideração todos os benefícios citados acima, é recorrente a análise de que a estratégia de expansão internacional exerce influência positiva sobre o desempenho das empresas. Nesse sentido, vários são os autores que conduziram estudos acerca dos efeitos da expansão internacional sobre o desempenho das empresas e encontraram uma relação linear positiva (Delios \& Beamish, 1999; Grant, 1987; Grant et al., 1988; Han et al., 1998).

Entretanto, esses autores têm sido alvo de críticas, devido ao fato de super valorizar os benefícios da expansão internacional e, por vezes, não considerar os custos inerentes ao processo de internacionalização. Para Ruigrok e Wagner (2003), a internacionalização, isoladamente, não é uma condição suficiente para um desempenho superior e, portanto, a empresa deve realizar uma avaliação entre os custos e os benefícios inerentes à estratégia de expansão internacional.

\section{Os custos da internacionalização}

Embora existam muitas considerações que levem a previsões de um relacionamento positivo entre o grau de internacionalização e o desempenho, diversos outros fatores levam a acreditar que possa haver também um impacto negativo do grau de internacionalização sobre a rentabilidade. Dentre esses fatores, destacam-se:

Restrições gerenciais: distância física, diferenças linguísticas e culturais, barreiras jurídicas (Siddharthan \& Lall, 1982);

Custo de ser estrangeiro (liability of foreignness): falta de informação local, falta de familiaridade com a cultura do lugar, tratamento discriminatório por parte do governo, clientes e fornecedores locais (Hymer, 1960; Li, 2007; Zaheer, 1995);

Custo de ser novo no mercado (liability of newness): instalação de fábricas no exterior, estabelecimento de sistemas internos de gestão e rede de negócios externos (Hymer, 1960; Li, 2007; Lu \& Beamish, 2004); 
Custos operacionais: fatores logísticos, barreiras comerciais (Capar \& Kotabe, 2003); custos de governança, coordenação e controle (Hitt, Hoskisson, \& Kim, 1997; Lu \& Beamish, 2004; Tallman $\& \mathrm{Li}, 1996)$

Fatores ambientais: regulamentações governamentais, leis de comércio e flutuações da moeda (Sundaram \& Black, 1992).

Levando em consideração os desafios e custos descritos acima, alguns estudos identificaram uma relação linear-negativa entre o grau de internacionalização e o desempenho da empresa (Brewer, 1981; Collins, 1990; Michel \& Shaked, 1986; Siddharthan \& Lall, 1982). Para Li (2007). Esses resultados podem estar associados ao fato de que, eventualmente, os custos marginais inerentes à expansão internacional podem exceder os benefícios marginais, afetando negativamente $o$ desempenho da própria empresa.

\section{$O$ interjogo entre benefícios e custos da internacionalização}

Os estudos mais recentes têm encontrado resultados não lineares para a relação entre o grau de internacionalização e o desempenho, indicando que as EMNs obtêm tanto benefícios quanto custos em seu processo de internacionalização (Chang, 2007; Contractor et al., 2003; Gomes \& Ramaswamy, 1999; Lu \& Beamish, 2004). Nesse sentido, Ruigrok e Wagner (2003) argumentam que "a internacionalização, por si só, não é uma condição suficiente para um desempenho superior, de modo que hipotetizar uma relação monotônica linear entre internacionalização-desempenho não consiste no melhor caminho à construção da teoria" (p. 68).

Para Gomes e Ramaswame (1999), é razoável que a relação internacionalização-desempenho seja não linear, podendo compreender 2 períodos: (a) período em que os benefícios da internacionalização tendem a exceder os custos da expansão internacional; e (b) momento em que os custos superam os benefícios incrementais da internacionalização. Adicionalmente, Li (2007) entende que "é plausível argumentar que a relação internacionalização-desempenho seja não linear, tendo em vista que o desempenho da empresa é determinado pelos efeitos combinados dos custos e benefícios das EMNs no curso da internacionalização" (p. 121).

Sendo assim, levando em consideração os benefícios e os custos inerentes ao processo de internacionalização, vários estudos encontraram um efeito não linear para a relação internacionalização-desempenho, entretanto, os seus resultados são bastante controversos. Há estudos em que os resultados foram em forma de $\mathbf{U}$, ou seja, em estágios iniciais de diversificação internacional, o desempenho apresentado foi negativo, mas com o grau de internacionalização aumentado, as empresas também tiveram melhor desempenho (Ruigrok \& Wagner, 2003). Existem ainda estudos cujos resultados foram em forma de $\mathbf{U}$ invertido (Daniels \& Bracker, 1989; Geringer et al., 1989; Gomes \& Ramaswamy, 1999), de modo que o desempenho subiu em estágios iniciais de internacionalização, mas caiu à medida em que aumentou o grau de internacionalização. Finalmente, Contractor, Kundu e Hsu (2003) encontraram uma relação curvilinear em forma de $\mathbf{S}$ horizontal, denominada teoria dos três estágios de internacionalização.

Seguindo comportamento semelhante aos resultados verificados em estudos com EMNs de economias desenvolvidas, observa-se que a relação internacionalização-desempenho no universo de EMNs de economias em desenvolvimento também tem considerado os custos e benefícios inerentes à estratégia de internacionalização: (a) relação em forma de $\mathbf{U}$ (Contractor, Kumar, \& Kundu, 2007; Thomas, 2006); (b) relação em forma de $\mathbf{S}$ horizontal (Chang, 2007); e (c) relação em forma de $\mathbf{U}$ invertido (H. Chen \& Hsu, 2010; Chiao \& Yang, 2011). Levando em consideração esses resultados, parece haver uma tendência de relação curvilinear entre o grau de internacionalização e o desempenho tanto em EMNs de economias desenvolvidas quanto em EMNs de economias em desenvolvimento. Sendo assim, a seguinte hipótese foi formulada: 
Hipótese da pesquisa: A relação entre o grau de internacionalização e desempenho em Empresas Multinacionais de economias em desenvolvimento será não linear, apresentando-se de forma quadrática (um ponto de inflexão) ou cúbica (dois pontos de inflexão).

\section{Aspectos Metodológicos}

\section{Amostra}

A amostra foi obtida a partir dos relatórios anuais, elaborados pela UNCTAD, nos anos de 2004 a 2009. Convém citar que cada relatório contém o ranking das 100 EMNs mais internacionalizadas de economias em desenvolvimento. Entretanto, a amostra do presente trabalho consiste de 33 EMNs (ver Tabela 1), devido ao fato de essas serem as empresas que estão presentes em todos os relatórios divulgados no período estudado de seis anos (2004-2009).

Tabela 1

\section{Relação das Empresas Multinacionais (EMNs) da Amostra}

\begin{tabular}{|c|c|c|c|c|}
\hline Ítem & Empresa & Fundação & Origem & Indústria \\
\hline 1 & Hutchison Whampoa Limited & 1999 & Hong Kong, China & Conglomerado \\
\hline 2 & CITIC Group & 1979 & China & Conglomerado \\
\hline 3 & Cemex S.A.B. de C.V. & 1906 & Mexico & Indústria de base \\
\hline 4 & Vale as & 1942 & Brazil & Indústria de base \\
\hline 5 & Samsung Electronics Co., Ltd. & 1969 & Korea, Republic of & Equips elétro-eletrônicos \\
\hline 6 & Hyundai Motor Company & 1967 & Korea, Republic of & Automobilístico \\
\hline 7 & LG Corp & 1958 & Korea, Republic of & Equips elétro-eletrônicos \\
\hline 8 & Singapore Telecommunications Ltd & 1879 & Singapore & Telecomunicações \\
\hline 9 & Formosa Plastics Group & 1954 & Taiwan Province of China & Químico \\
\hline 10 & Petroleo Brasileiro as & 1953 & Brazil & Indústria de base \\
\hline 11 & MTN Group Ltd & 1994 & South Africa & Telecomunicações \\
\hline 12 & Gerdau as & 1901 & Brazil & Indústria de base \\
\hline 13 & China National Petroleum Corporation & 1988 & China & Indústria de base \\
\hline 14 & América Móvil SAB de CV & 2000 & Mexico & Telecomunicações \\
\hline 15 & Noble Group Ltd & 1987 & Hong Kong, China & Conglomerado \\
\hline 16 & Flextronics International Ltd & 1969 & Singapore & Equips elétro-eletrônicos \\
\hline 17 & Oil and Natural Gas Corp Ltd & 1955 & India & Indústria de base \\
\hline 18 & CapitaLand Ltd & 2000 & Singapore & Conglomerado \\
\hline 19 & New World Development Ltd & 1970 & Hong Kong, China & Conglomerado \\
\hline 20 & CLP Holdings Ltd & 1901 & Hong Kong, China & Utilidades \\
\hline 21 & Sinochem Group & 1950 & China & Indústria de base \\
\hline 22 & Shangri-La Asia Ltd & 1971 & Hong Kong, China & Serviços \\
\hline 23 & Sasol Limited & 1950 & South Africa & Químico \\
\hline 24 & Quanta Computer Inc & 1988 & Taiwan Province of China & Equips elétro-eletrônicos \\
\hline
\end{tabular}


Tabela 1 (continuação)

\begin{tabular}{lllll}
\hline Ítem & Empresa & Fundação & Origem & Indústria \\
\hline 25 & Li \& Fung Ltd & 1906 & Hong Kong, China & Serviços \\
26 & Naspers Ltd & 1915 & South Africa & Serviços \\
27 & Acer Inc & 1976 & Taiwan Province of China & Equips elétro-eletrônicos \\
28 & Steinhoff International Holdings Ltd & 1965 & South Africa & Serviços \\
29 & Fomento Economico Mexicano SAB & 1890 & Mexico & Alitos, bebs, tab \\
30 & Fraser and Neave Ltd & 1883 & Singapore & Alitos, bebs, tab \\
31 & Gold Fields Ltd & 1998 & South Africa & Indústria de base \\
32 & Sappi Ltd & 1936 & South Africa & Indústria de base \\
33 & Taiwan Semiconductor Manufacturing Co Ltd & 1987 & Taiwan Province of China & Equips elétro-eletrônicos \\
\hline
\end{tabular}

Nota. Fonte: elaborado pelos autores adaptado da United Nations Conference on Trade and Development. (2009). Transnational corporations, agricultural production and development (World Investment Report/2009), New York and Geneva, Division on Investment and Enterprise, United Nations; United Nations Conference on Trade and Development. (2008). Transnational corporations and the infrastructure challenge (World Investment Report/2008), New York and Geneva, Division on Investment and Enterprise, United Nations; United Nations Conference on Trade and Development. (2007). Transnational corporations, extractive industries and development (World Investment Report/2007), New York and Geneva, Division on Investment and Enterprise, United Nations; United Nations Conference on Trade and Development. (2006). FDI from developing and transition economies: implications for development (World Investment Report/2006), New York and Geneva, Division on Investment and Enterprise, United Nations; United Nations Conference on Trade and Development. (2005). Transnational corporations and the internationalization of $R \& D$ (World Investment Report/2005), New York and Geneva, Division on Investment and Enterprise, United Nations; United Nations Conference on Trade and Development. (2004). The shift towards services (World Investment Report/2004 New York and Geneva, Division on Investment and Enterprise, United Nations.

\section{Modelo de pesquisa} estudo.

A Figura 1 apresenta uma visão geral do modelo hipotético e conceitual que norteia o presente

\section{EMNs \\ GRAU DE INTERNACIONALIZAÇÃO}

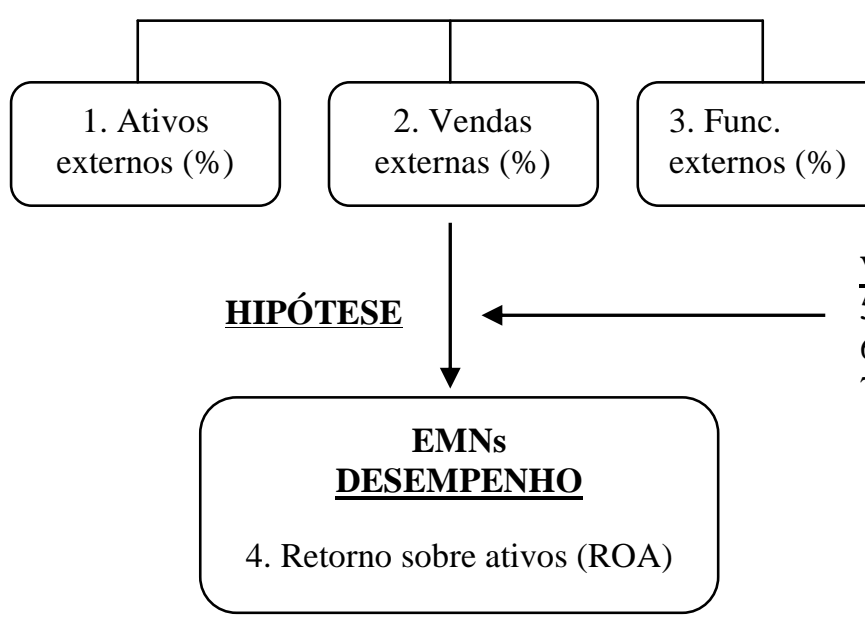

VARIÁVEIS DE CONTROLE

5. Tamanho da EMN;

6. Efeitos da indústria; e

7. País de origem.
1. Valor dos ativos no exterior / valor total dos ativos da empresa;

2. Receita bruta de subsidiárias no exterior / receitas totais;

3. Número de funcionários no exterior / número de funcionários total;

4. Lucro líquido / ativos totais;
5. Logaritmo natural do número total de funcionários;

6. Variáveis dummy de 9 indústrias;

7. País de origem da EMN.

Figura 1. Modelo Hipotético e Conceitual. 
Como pode ser visto na Figura 1, a variável independente, grau de internacionalização, é formada por três outras variáveis: (a) a razão dos ativos externos em relação aos ativos totais da empresa; (b) a razão das vendas externas em relação às vendas totais da empresa; e (c) a razão dos funcionários externos em relação aos funcionários totais. Assim, essas três variáveis formam juntas o construto grau de internacionalização. Por outro lado, a variável dependente do modelo, desempenho, é mensurada pelo retorno sobre os ativos ou Return on Assets (ROA) das EMNs. Adicionalmente, de modo a atingir um melhor ajustamento do modelo, foram estabelecidas três variáveis de controle: (a) o tamanho da empresa; (b) os efeitos da indústria; e (c) a região de origem da empresa.

\section{Construção das variáveis}

\section{Variável independente: grau de internacionalização}

A grande maioria dos estudos anteriores utilizaram o indicador Foreign Sales to Total Sales (FSTS), que consiste na proporção das vendas externas em relação às vendas totais, para indicar o grau de diversificação internacional ou o nível de transnacionalidade das empresas multinacionais (Capar \& Kotabe, 2003; Grant, 1987; Habib \& Victor, 1991; Tallman \& Li, 1996). Entretanto, não há consenso entre os autores sobre a efetividade dessa variável, quando utilizada de forma isolada (Gomes \& Ramaswamy, 1999). Annavarjula e Beldona (2000) fizeram uma revisão e reconceitualização dos estudos anteriores sobre a relação entre o grau de internacionalização e o desempenho, através da qual encontraram que a variável independente, o grau de internacionalização, tem sido conceituada de quatro modos diferentes na literatura: (a) controle: os autores que seguem essa conceituação associam a internacionalização ao controle e a coordenação global de ativos de uma empresa e atividades relacionadas; (b) diversificação: a internacionalização é associada aos segmentos geográficos que são atendidos pela empresa; (c) operações: volume de produção, vendas e P\&D no exterior; e (d) orientação: está relacionada com a disposição e a atitude de uma empresa em um contexto internacional.

Por outro lado, Wagner e Ruigrok (2004), em sua meta-análise dos estudos anteriores sobre a relação entre o grau de internacionalização e o desempenho, encontraram que a variável independente, grau de internacionalização, tem sido conceitualizada pelos autores de três modos distintos:

1. Estrutural: define a internacionalização em termos da proporção de ativos externos em relação aos ativos totais (Severn \& Laurence, 1974), número de subsidiárias (Delios \& Beamish, 1999; Lu \& Beamish, 2001, 2004; Morck \& Yeung, 1991); e proporção de funcionários localizados no exterior em relação aos funcionários totais;

2. Financeiro: define internacionalização em termos das vendas externas em relação às vendas totais (Capar \& Kotabe, 2003; Collins, 1990; Geringer et al., 1989; Grant, 1987; Kotabe, Srinivasan, \& Aulakh, 2002; Li, 2005; Michel \& Shaked, 1986; Qian, 1998; Qian, 2002; Ruigrok \& Wagner, 2003; Siddharthan \& Lall, 1982), lucros externos em relação aos lucros totais; e

3. Índices: define o grau de internacionalização como um índice combinado de duas ou mais medidas, podendo compreender os dois modos anteriores.

$\mathrm{Na}$ literatura de internacionalização-desempenho, há um consenso de que a mensuração da internacionalização por meio de um índice composto tende a ser mais robusta, por apresentar menor erro de predição e, portanto, ser capaz de alcançar maior validade enquanto construto (Annavarjula \& Beldona, 2000; Gomes \& Ramaswamy, 1999; Sullivan, 1994; Wagner \& Ruigrok, 2004). Por isso, o presente estudo utilizou o Transnationality Index (TNI) ou índice de transnacionalização, proposto pela United Nations Conference on Trade and Development UNCTAD, para a mensuração do Grau de Internacionalização das EMNs de economias em desenvolvimento.

O Transnationality Index (TNI) propõe que o grau de internacionalização das empresas deve ser calculado como a média aritmética dos seguintes indicadores de vendas, produção e recursos: (a) 
proporção das vendas externas em relação às vendas totais; (b) proporção dos ativos estrangeiros em relação aos ativos totais; e (c) proporção do número de funcionários no exterior em relação ao total de funcionários. Para melhor visualização, a Figura 1 demonstra as variáveis que compõem o construto de diversificação internacional ou grau de internacionalização que foi utilizado como variável independente no modelo estatístico deste estudo.

\section{Variável dependente: desempenho}

A variável dependente foi representada pelo retorno sobre os ativos ou Return on Assets (ROA) para medir o desempenho das EMNs, semelhantemente ao que foi feito em vários estudos anteriores sobre a relação internacionalização-desempenho (C. W. Kim, Hwang, \& Burgers, 1989; Grant et al., 1988; Haar, 1989; Habib \& Victor, 1991; Hoskisson, 1987). O retorno sobre os ativos é um indicador que reflete a rentabilidade de uma empresa em relação ao seu total de ativos, indicando o quanto a gestão é eficiente na utilização do seu ativo total. Portanto, tal medida vai ao encontro dos objetivos deste estudo.

\section{Variáveis de controle: tamanho da empresa, efeitos da indústria e região de origem da empresa}

Em consonância com estudos anteriores, o presente trabalho estabeleceu as seguintes variáveis de controle: o tamanho da empresa (assim como Capar \& Kotabe, 2003; Chang, 2007; Contractor et al., 2003; Gaur \& Kumar, 2009; Grant, 1987; Riahi-Belkaoui, 1998; Ruigrok \& Wagner, 2003); os efeitos da indústria (também utilizado por Capar \& Kotabe, 2003; Chang, 2007; Gaur \& Kumar, 2009; Ruigrok \& Wagner, 2003); e o país de origem da empresa (como Li, 2005). O tamanho da empresa foi mensurado como o logaritmo natural do total de funcionários (assim como em Bobillo, LópesIturriaga, \& Tejerina-Gaite, 2010; Capar \& Kotabe, 2003; Chang, 2007; Contractor et al., 2003; Goerzen \& Beamish, 2003). Os efeitos da indústria foram representados por um conjunto de 9 variáveis dummy $(\mathrm{I} 1=$ conglomerados; $\mathrm{I} 2=$ indústrias de base, $\mathrm{I} 3=$ equipamentos elétrico-eletrônicos, $\mathrm{I} 4=$ automobilística, $\mathrm{I} 5=$ telecomunicações, $\mathrm{I} 6=$ utilidades, $\mathrm{I} 7=$ serviços, $\mathrm{I} 8=$ químico, $\mathrm{I} 9=$ alimentos, bebidas e tabaco).

Para melhor visualização, a Tabela 2 resume todas as variáveis do modelo hipotético conceitual do estudo e descreve suas respectivas fontes.

Tabela 2

Definição das Variáveis e Respectivas Fontes

\begin{tabular}{|c|c|c|}
\hline Variável & Definição & Estudo base (Fonte) \\
\hline \multicolumn{3}{|l|}{ Variáveis independentes } \\
\hline $\begin{array}{l}\text { GIO - Grau de Internacionalização } \\
\text { de Operações }\end{array}$ & $\begin{array}{l}\text { Proporção das vendas, ativos e } \\
\text { funcionários externos em relação ao } \\
\text { total. }\end{array}$ & Metodologia da UNCTAD. \\
\hline \multicolumn{3}{|l|}{ Variável dependente } \\
\hline DES - Desempenho & Retorno sobre os ativos (ROA). & $\begin{array}{l}\text { Hoskisson (1987); Grant et al. } \\
\text { (1988); Haar (1989); C. W. Kim et } \\
\text { al. (1989); Habib e Victor (1991). }\end{array}$ \\
\hline \multicolumn{3}{|l|}{ Variáveis de controle } \\
\hline TAM - Tamanho da EMN & $\begin{array}{l}\text { Logaritmo natural do total de } \\
\text { funcionários da empresa. }\end{array}$ & $\begin{array}{l}\text { Chang (2007); Contractor et al. } \\
\text { (2003); Goerzen e Beamish (2003); } \\
\text { Capar e Kotabe (2003); Bobillo et } \\
\text { al. (2010). }\end{array}$ \\
\hline
\end{tabular}


Tabela 2 (continuação)

\begin{tabular}{lll}
\hline Variável & Definição & Estudo base (Fonte) \\
\hline PAÍS DE ORIGEM & País de origem da EMN. & Li (2005) \\
Efeitos da indústria & Conjunto de variáveis dummy. & $\begin{array}{l}\text { Hoskisson (1987); Grant et al. } \\
\text { (1988); Contractor } \text { et al. }(2003) ; \\
\text { Chang (2007); Gaur e Kumar } \\
\text { (2009). }\end{array}$ \\
$I_{1}=$ Conglomerados & \\
$I_{2}=$ Indústrias de base & \\
$I_{3}=$ Equipamentos elétrico- & \\
eletrônicos & & \\
$I_{4}=$ Automobilística & \\
$I_{5}=$ Telecomunicações & \\
$I_{6}=$ Utilidades & \\
$I_{7}=$ Serviços & \\
$I_{8}=$ Químico & \\
$I_{9}=$ Alimentos, bebidas e tabaco & \\
\hline
\end{tabular}

\section{Coleta de dados}

A coleta de dados foi realizada em duas fontes: (a) UNCTAD e (b) Thomson Reuters. A variável independente do modelo (grau de internacionalização) foi obtida a partir dos rankings do período 2004-2009 das empresas multinacionais (EMNs) mais internacionalizadas de economias em desenvolvimento, elaborado pela UNCTAD. Tais rankings são construídos a partir das informações financeiras das empresas relativas ao ano fiscal, cujo período corresponde a 01/04 a 31/03 de cada ano analisado. No que diz respeito à variável dependente do modelo (desempenho das operações), as informações foram obtidas a partir da base de dados Thomson One pertencente à Thomson Reuters, que dispõe de demonstrativos financeiros de empresas listadas em bolsas de valores ao redor do mundo. A variável desempenho, medida pelo Return on Assets (ROA), também foi coletada para o período de 2004-2009. Assim, um conjunto de dados foi obtido, com $\mathbf{N}$ empresas, sendo que, para cada uma dessas, há um número $\mathbf{T}$ de observações ao longo do tempo, de modo que o número total de observações se dá através de N x T. Ou seja, 33 x $6=198$ observações, configurando na formação de um conjunto de dados de corte transversal ( 33 empresas) associados a uma série de tempos (6 anos).

\section{Técnicas estatísticas}

Para analisar a relação internacionalização-desempenho e testar a hipótese do estudo, este trabalho utilizou duas técnicas de análise: (a) Regressão Múltipla (MQO), procedimento adotado anteriormente em vários estudos (Capar \& Kotabe, 2003; Chang, 2007; Goerzen \& Beamish, 2003; Lu \& Beamish, 2004; Tallman \& Li, 1996); e (b) Análise de Dados em Painel, técnica que permite uma análise das variações das unidades transversais simultaneamente com as variações das unidades individuais ao longo do tempo. A maior parte dos estudos anteriores utiliza apenas a técnica de regressão múltipla (MQO), entretanto, este estudo utilizará também a técnica de dados em painel, de modo a tornar as estimativas mais eficientes. Para Hsiao (2003), uma das principais vantagens em se utilizar dados em painel é que, geralmente, eles dão ao pesquisador um grande número de pontos de dados, aumentando os graus de liberdade e reduzindo a colinearidade entre as variáveis explicativas, melhorando assim a eficiência das estimativas. 


\section{Análise de regressão múltipla (mínimos quadrados ordinários-mqo) e análise de dados em painel}

O trabalho examinou os efeitos do grau de internacionalização sobre o desempenho das 33 EMNs, através da Análise de Regressão Múltipla, por meio do método dos Mínimos Quadrados Ordinários (MQO) ou Ordinary least squares (OLS), de modo a maximizar o nível de ajustamento do modelo aos dados observados no período 2004-2009.

Para testar a hipótese do trabalho, os três modelos ou equações de regressão descritos na Tabela 3 foram aplicados aos dados com o objetivo de avaliar qual deles melhor se ajusta à relação existente entre o grau de internacionalização e o desempenho das EMNs da amostra. Em outras palavras, a hipótese será aceita caso os modelos não lineares quadrático ou cúbico sejam mais ajustados aos dados da amostra do que a equação (1), indicando uma relação curvilinear entre o grau de internacionalização e o desempenho. De forma específica, foi realizada uma análise comparativa entre o efeito linear versus o efeito curvilinear quadrático e cúbico da estratégia de expansão internacional sobre o desempenho das EMNs da amostra.

Tabela 3

\section{Descrição dos Três Modelos Testados na Pesquisa através do Método dos Mínimos Quadrados Ordinários (MQO)}

MODELO

Regressão:

(1) Modelo linear:

$\mathrm{DES}=\beta_{0}+\beta_{1} \mathrm{GIO}+\beta_{2} \mathrm{TAM}+\beta_{3} \mathrm{I}_{1}+\beta_{4} \mathrm{I}_{2}+\beta_{5} \mathrm{I}_{3}+\beta_{6} \mathrm{I}_{4}+$ $\beta_{7} \mathrm{I}_{5}+\beta_{8} \mathrm{I}_{6}+\beta_{9} \mathrm{I}_{7}+\beta_{10} \mathrm{I}_{8}+\beta_{11} \mathrm{I}_{9}+\beta_{12} \mathrm{REG}_{1}+\beta_{13} \mathrm{REG}_{2}+$ $\beta_{14} \mathrm{REG}_{3}+\varepsilon$

(2) Modelo não linear (quadrático):

$\mathrm{DES}=\beta_{0}+\beta_{1} \mathrm{GIO}+\beta_{2} \mathrm{GIO}^{2}+\beta_{3} \mathrm{TAM}+\beta_{4} \mathrm{I}_{1}+\beta_{5} \mathrm{I}_{2}+\beta_{6}$ $\mathrm{I}_{3}+\beta_{7} \mathrm{I}_{4}+\beta_{8} \mathrm{I}_{5}+\beta_{9} \mathrm{I}_{6}+\beta_{10} \mathrm{I}_{7}+\beta_{11} \mathrm{I}_{8}+\beta_{12} \mathrm{I}_{9}+\beta_{13} \mathrm{REG}_{1}+$ $\beta_{14} \mathrm{REG}_{2}+\beta_{15} \mathrm{REG}_{3}+\varepsilon$

(3) Modelo não-linear (cúbico):

$\mathrm{DES}=\beta_{0}+\beta_{1} \mathrm{GIO}+\beta_{2} \mathrm{GIO}^{2}+\beta_{3} \mathrm{GIO}^{3}+\beta_{4} \mathrm{TAM}+\beta_{5} \mathrm{I}_{1}+$ $\beta_{6} \mathrm{I}_{2}+\beta_{7} \mathrm{I}_{3}+\beta_{8} \mathrm{I}_{4}+\beta_{9} \mathrm{I}_{5}+\beta_{10} \mathrm{I}_{6}+\beta_{11} \mathrm{I}_{7}+\beta_{12} \mathrm{I}_{8}+\beta_{13} \mathrm{I}_{9}+$ $\beta_{14} \mathrm{REG}_{1}+\beta_{15} \mathrm{REG}_{2}+\beta_{16} \mathrm{REG}_{3}+\varepsilon$
TESTE DE HIPÓTESE

O modelo não linear quadrático - com um ponto de inflexão - será apoiado caso os indicadores: $\mathrm{R}^{2}, \mathrm{R}^{2}$ ajustado e Teste $\mathrm{F}$ associados à equação (2) sejam significantemente maiores do que os indicadores das equações (1) e (3), bem como o coeficiente do termo quadrado do grau de internacionalização, variável $\beta_{2}$ (equação 2), seja significante.

O modelo não linear cúbico - com dois pontos de inflexão - será apoiado caso os indicadores: $\mathrm{R}^{\mathbf{2}}, \mathrm{R}^{\mathbf{2}}$ ajustado e Teste F associados à equação (3) sejam significantemente maiores do que os indicadores das equações (1) e (2), bem como o coeficiente do termo cúbico do grau de internacionalização, variável $\beta_{3}$ (equação 3), seja significante.

Em que:

$\boldsymbol{D E S}=$ Desempenho financeiro calculado pela taxa de retorno sobre ativos; $\boldsymbol{T A} \boldsymbol{M}=$ Tamanho da empresa, representado pelo logaritmo natural do total de funcionários; $\boldsymbol{G I O}=$ Grau de internacionalização das operações; $\boldsymbol{G I O}^{2}=$ Termo quadrado do grau de internacionalização das operações; $\boldsymbol{G I O}^{3}=$ Termo cúbico do grau de internacionalização das operações; $\boldsymbol{I}_{i}=$ Conjunto de variáveis dummy para controlar os efeitos do setor; $\boldsymbol{R E} \boldsymbol{G}=$ Variáveis dummy para controlar os efeitos do país de origem; $\varepsilon=$ erro.

No que diz respeito à análise de dados em painel, o presente estudo fez uma análise comparativa entre os efeitos fixos e os efeitos aleatórios de modo a indicar qual se ajusta melhor aos dados. Para tanto, realizou-se o teste de Hausman, que permite realizar uma comparação entre os estimadores de efeitos fixos e aleatórios (Hsiao, 2003). O teste de Hausman verifica a hipótese nula de que os coeficientes esperados pelo estimador dos efeitos aleatórios eficientes são os mesmos que o esperado pelo estimador dos efeitos fixos consistentes. Se o P-valor do teste não possuir significância $(\mathrm{P}>0,05)$, então é seguro usar efeitos aleatórios. No entanto, se o P-valor do teste tiver significância $(\mathrm{P}<0,05)$, então deverão ser usados efeitos fixos. 


\section{Apresentação e Discussão dos Resultados da Pesquisa}

\section{Análise descritiva dos resultados e atendimento aos pressupostos da Regressão Múltipla}

A Tabela 4 apresenta as correlações das variáveis independentes utilizadas no estudo, sendo que os resultados não revelam qualquer problema de forte multicolinearidade, tendo em vista que as variáveis independentes não são altamente correlacionadas entre si (Hair, Anderson, Tatham, \& Black, 1998). Além disso, foram feitas estatísticas Tolerance e Variance Inflation Factor (VIF), sendo que não foram detectados, através desses testes, problemas de multicolinearidade. Os dados atenderam ainda a outros três pressupostos da regressão: (a) ausência de autocorrelação serial (Teste DurbinWatson $=1,766$ ) para o modelo 1 , que melhor se ajustou aos dados; (b) normalidade (Teste Kolmogorov-Smirnov/Sig. = 0,150 (P>0,05); e (c) homoscedasticidade (Teste de Pesaran-Pesaran/Sig. $=0,151(\mathrm{P}>0,05)$. Por fim, para validar os resultados da regressão múltipla, a amostra coletada foi repartida aleatoriamente em duas subamostras (uma com 51,5\% dos casos; a outra com 48,5\% dos casos), de modo que cada uma delas foi processada de forma independente e os seus resultados comparados entre si. Verificou-se, então, que os dados se comportaram de forma razoavelmente homogênea em relação à amostra geral, não se verificando parâmetros $\left(R^{2}, R^{2}\right.$ ajustado, Teste $F$, Durbin-Watson, GIO) que destoassem de forma acentuada.

Tabela 4

Médias, desvio padrão e correlações $(\mathrm{N}=33 * 6=198)$

\begin{tabular}{lrrrrr}
\hline Variáveis & Média & D.P & $\mathbf{1}$ & $\mathbf{2}$ & $\mathbf{3}$ \\
\hline 1. Grau de Internacionalização (GIO) & 0,47 & 0,22 & 1.00 & & \\
2. Desempenho (DES) & 0,09 & 0,05 & $-0,229^{* *}$ & 1.00 & \\
3. Tamanho (TAM) & 10,49 & 1,18 & $-0,346^{* * *}$ & $-0,198^{* *}$ & 1.00 \\
\hline
\end{tabular}

Nota. $*<0.05 ; * *<0.01 ; * * *<0.001$.

A Figura 2 apresenta um diagrama de dispersão das 198 observações para as variáveis desempenho e grau de internacionalização das EMNs. A partir de uma análise descritiva, verifica-se que, à medida que o grau de internacionalização aumenta, há uma tendência de diminuição do desempenho. Para avaliação mais detalhada desse comportamento, utilizamos as médias de desempenho por intervalos de internacionalização (Tabela 5).

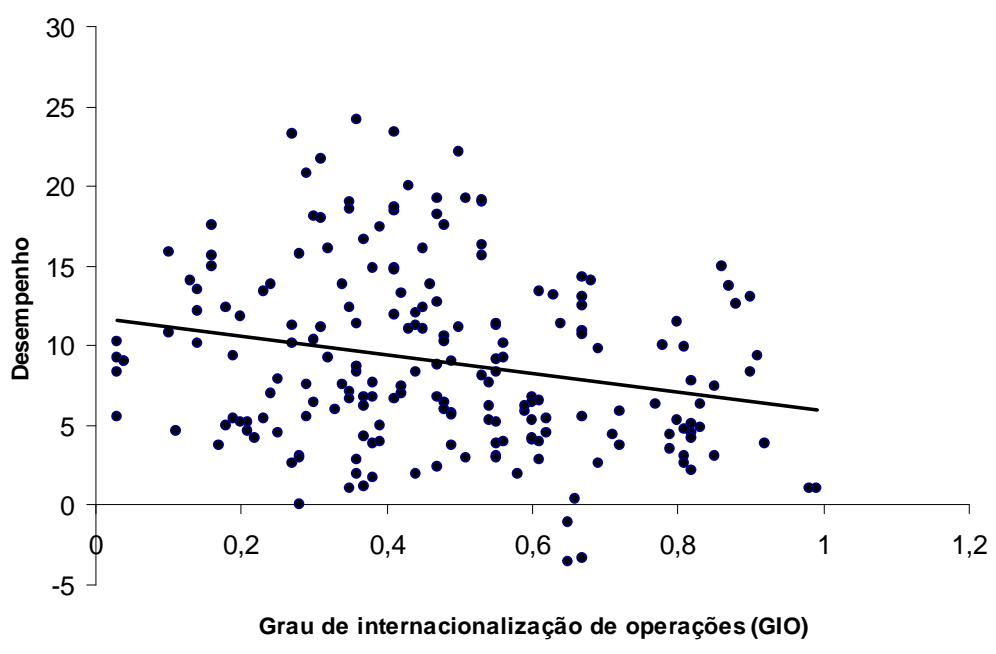

Figura 2. Dispersão dos Dados ( $=198 ; 33$ empresas para o período 2004-2009). 
A comparação de médias foi aplicada em uma escala de quatro intervalos de internacionalização (1-39,9\%; 40-59,9\%; 60-79,9\%; 80-99,9\%), procedimento utilizado anteriormente por Ruigrok e Wagner (2003). Tais intervalos foram definidos a partir da média do Transnationality Index (TNI) ou do grau de internacionalização das EMNs da amostra.

Tabela 5

Evolução do Desempenho das EMNs segundo a Variação do Grau de Internacionalização (Comparação de Médias)

\begin{tabular}{lllll}
\cline { 2 - 4 } & \multicolumn{4}{l}{ Grau de internacionalização de operações (GIO) } \\
\hline Medida de desempenho & Estágio 1 & Estágio 2 & Estágio 3 & Estágio 4 \\
& $(0,1-39,9 \%)$ & $(40-59,9 \%)$ & $(60-79,9 \%)$ & $(80-99,9 \%)$ \\
\cline { 1 - 4 } Retorno sobre Ativos (ROA) & $9,6 \%$ & $10,5 \%$ & $6,4 \%$ & $6,7 \%$ \\
\hline
\end{tabular}

Em consistência com os resultados obtidos no diagrama de dispersão, bem como na análise de regressão múltipla (parte 'Análise dos resultados da regressão múltipla (MQO) e dados em painel' do trabalho), verifica-se um comportamento decrescente do desempenho, quando analisada a sua evolução através do primeiro ao quarto estágio de internacionalização (Figura 3).

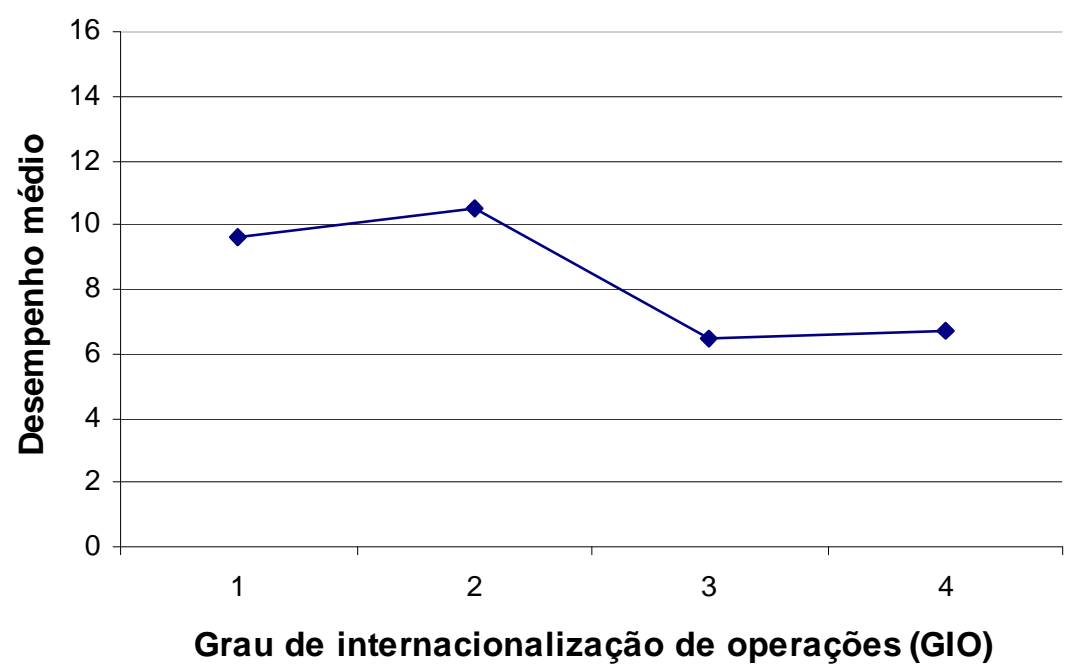

Figura 3. Figura do Desempenho através dos Quatro Estágios de Internacionalização.

Em outras palavras, as estatísticas descritivas sugerem que quanto maior o grau de internacionalização, menor tende a ser o desempenho das EMNs de economias em desenvolvimento. Entretanto, a parte a seguir apresenta uma melhor avaliação da relação internacionalizaçãodesempenho para as EMNs de economias em desenvolvimento.

\section{Análise dos resultados da regressão múltipla (MQO) e dados em painel}

A Tabela 6 apresenta os resultados dos três modelos que foram testados: modelo linear, modelo não linear quadrático e modelo não linear cúbico da relação entre o grau de internacionalização e desempenho das EMNs de economias em desenvolvimento.

O primeiro modelo na Tabela 6 é um exame do efeito linear da expansão internacional sobre o desempenho (ROA). Como pode ser visto, há uma relação estatisticamente significativa negativa entre o grau de internacionalização e o desempenho, ao nível de significância de $P<0.001$. Esse resultado é consistente com resultados de outros estudos (Brewer, 1981; Collins, 1990; Michel \& Shaked, 1986; Siddharthan \& Lall, 1982). Além disso, o modelo geral também é significante $\left(P<0.001, \mathrm{R}^{2} 0,430\right)$. 
Dentre as variáveis de controle, destaca-se o tamanho da empresa que foi estatisticamente significante $(P<0.001)$ e apresentou um coeficiente de inclinação negativa. Em outras palavras, observou-se certa tendência de que quanto maior o tamanho da empresa (em termos do número total de funcionários), menor o desempenho da EMN (em termos de ROA), assumindo que o nível de diversificação internacional seja constante. Esse resultado é consistente com os resultados obtidos por Capar e Kotabe (2003).

O segundo modelo na Tabela 6 é um exame do efeito não linear quadrático - um ponto de inflexão - da expansão internacional sobre o desempenho (ROA). Como pode ser observado, não há apoio para a hipótese de que existe uma relação não linear quadrática entre o grau de internacionalização e o desempenho, uma vez que o termo quadrado do grau de internacionalização, $\mathrm{GIO}^{2}$, não é significante. Adicionalmente, verifica-se que a inclusão do $\mathrm{GIO}^{2}$ não melhorou de forma consistente o modelo geral, uma vez que o $\mathrm{R}^{2}$ ajustado passou de 0,430 para 0,431 , e o Teste $\mathrm{F}$ foi também reduzido de $(\mathrm{F}=13.406 ; P<0.001)$ para $(\mathrm{F}=12.477 ; P<0.001)$.

Tabela 6

Efeitos do Grau de Internacionalização sobre o Desempenho das EMNs

\begin{tabular}{|c|c|c|c|c|}
\hline \multirow[t]{2}{*}{ Variáveis independentes } & \multicolumn{3}{|c|}{ Método dos Mínimos Quadrados Ordinários (MQO) } & \multirow{2}{*}{$\begin{array}{r}\text { Análise de dado } \\
\text { em Paine } \\
\text { 4. Efeitos fixos }\end{array}$} \\
\hline & 1. Modelo linear & $\begin{array}{l}\text { 2. Modelo } \\
\text { quadrático }\end{array}$ & 3. Modelo cúbico & \\
\hline Intercepto & $31,438 * * *$ & $33,948 * * *$ & $31,724 * * *$ & $26,766 * * *$ \\
\hline GIO & $-0,488 * * *$ & $-0,777 * *$ & $-0,238$ & $-13,316^{* * *}$ \\
\hline$G I O^{2}$ & & 0,286 & $-0,984$ & \\
\hline$G I O^{3}$ & & & 0,764 & \\
\hline$T A M$ & $-0,352 * * *$ & $-0,378 * * *$ & $-0,360 * * *$ & $-2,006 * * *$ \\
\hline$I_{1}$ & $-0,122$ & $-0,103$ & $-0,119$ & 2,947 \\
\hline$I_{2}$ & & & & $5,410 * * *$ \\
\hline$I_{3}$ & 0,032 & 0,068 & 0,049 & $5,957 * * *$ \\
\hline$I_{4}$ & $-0,204 * * *$ & $-0,193 * *$ & $-0,207 * *$ & $-3,219$ \\
\hline$I_{5}$ & $0,359 * * *$ & $0,376 * * *$ & $0,379 * * *$ & $12,585^{* * *}$ \\
\hline$I_{6}$ & $-0,077$ & $-0,072$ & $-0,081$ & $4,4984 *$ \\
\hline$I_{7}$ & $0,231 * * *$ & $0,232 * *$ & $0,220 * *$ & $10,544 * * *$ \\
\hline$I_{8}$ & $0,203 * * *$ & $0,217 * * *$ & $0,199 * *$ & $11,970 * * *$ \\
\hline$I_{9}$ & $-0,195 * * *$ & $-0,182 * *$ & $-0,187 * *$ & \\
\hline Região de origem 1 & & & & $3,761 * * *$ \\
\hline Região de origem 2 & $0,205^{* *}$ & $0,231 * *$ & $0,212 * *$ & $5,822 * * *$ \\
\hline Região de origem 3 & $-0,183 * *$ & $-0,159^{*}$ & $-0,167 *$ & \\
\hline $\mathrm{R}^{2}$ & 0,465 & 0,469 & 0,471 & 0,601 \\
\hline $\mathrm{R}^{2}$ ajustado & 0,430 & 0,431 & 0,430 & 0,487 \\
\hline Teste F & $13,406^{* * *}$ & $12,477 * * *$ & $11,623 * * *$ & $5,243 * * *$ \\
\hline $\mathrm{N}^{\mathrm{o}}$ de observações $(33 \times 6)$ & 198 & 198 & 198 & 198 \\
\hline
\end{tabular}


Tabela 6 (continuação)

\begin{tabular}{|c|c|c|c|c|}
\hline \multirow[t]{2}{*}{ Variáveis independentes } & \multicolumn{3}{|c|}{ Método dos Mínimos Quadrados Ordinários (MQO) } & \multirow{2}{*}{$\begin{array}{r}\text { Análise de dados } \\
\text { em Painel } \\
\text { 4. Efeitos fixos }\end{array}$} \\
\hline & 1. Modelo linear & $\begin{array}{l}\text { 2. Modelo } \\
\text { quadrático }\end{array}$ & 3. Modelo cúbico & \\
\hline
\end{tabular}

\section{Definições:}

GIO:

Grau de internacionalização de operações

$\mathrm{GIO}^{2}$ :

Termo quadrado do grau de internacionalização de operações

$\mathrm{GIO}^{3}$ :

Termo cúbico do grau de internacionalização de operações

TAM:

Tamanho da EMN

Ii:

Efeitos do tipo de indústria: variável dummy 1-9. Nos modelos 1, 2 e 3, a indústria de base, $I 2$, foi excluída devido a problemas de colinearidade exata, identificados pelo SPSS. No modelo 4, o mesmo aconteceu para a indústria de alimentos, bebidas e tabaco, 19 .

Indústrias consideradas no modelo:

1. Conglomerados 6. Utilidades

2. Indústrias de 7. Serviços

base

3. Equipamentos 8. Setor Químico eletro-eletrônicos

4. Automobilístico 9. Alimentos, bebidas e tabaco

5. Telecomunicações

Origem:

Região de origem da EMN: variável dummy 1-3. Nos modelos 1, 2 e 3, a região de origem 1 foi excluída, devido a problemas de colinearidade exata, identificados pelo SPSS. No modelo 4, o mesmo aconteceu para a região de origem 3.

Origem 1: Ásia

Origem 3: África do Sul

Origem 2: América

do Sul

\section{Notas explicativas:}

(1).

A significância dos coeficientes de regressão e teste $\mathrm{F}$ é descrita através dos símbolos *, ** e ***, conforme segue:

$$
\begin{array}{r}
*<0.05 \\
* *<0.01 \\
* * *<0.001
\end{array}
$$

Os modelos foram testados com os dados relativos a 33 EMNs para um período de 6 anos (2004-2009), procedendo-se com uma análise empilhada ou Pooled analysis, através das técnicas de MQO e dados em painel.

MQO: Coeficientes padronizados; Painel Efeitos Fixos: Coeficientes não padronizados.

SPSS utilizado para as análises MQO (modelos 1, 2 e 3); GRETL utilizado para as análises de dados em Painel (modelo 4). 


\section{Tabela 6 (continuação)}

\begin{tabular}{|c|c|c|c|c|}
\hline \multirow[t]{2}{*}{ Variáveis independentes } & \multicolumn{3}{|c|}{ Método dos Mínimos Quadrados Ordinários (MQO) } & \multirow{2}{*}{$\begin{array}{r}\text { Análise de dados } \\
\text { em Painel } \\
\text { 4. Efeitos fixos }\end{array}$} \\
\hline & 1. Modelo linear & $\begin{array}{l}\text { 2. Modelo } \\
\text { quadrático }\end{array}$ & 3. Modelo cúbico & \\
\hline$(5)$ & \multicolumn{4}{|c|}{$\begin{array}{l}\text { Teste de Hausman: qui-quadrado }=38,2517 ; \mathrm{P} \text {-valor }=0,000139668 \text {. Portanto, } \\
\text { como o P-valor do teste possui significância }(\mathrm{P}<0,05) \text {, então foram utilizados } \\
\text { efeitos fixos (modelo } 4) .\end{array}$} \\
\hline (6). & \multicolumn{4}{|c|}{$\begin{array}{l}\text { Teste de Breusch-Pagan: qui-quadrado }=1,50765 ; \mathrm{P} \text {-valor }=0,219498 \text {. Portanto, } \\
\text { como o P-valor do teste não possui significância }(\mathrm{P}>0,05) \text {, então se deve utilizar o } \\
\text { modelo por MQO, pois não há efeitos específicos das empresas que comprometam } \\
\text { a utilização desse método. }\end{array}$} \\
\hline
\end{tabular}

O terceiro modelo na Tabela 6 é um exame do efeito não linear cúbico - dois pontos de inflexão - da expansão internacional sobre o desempenho (ROA). Como pode ser visto, não há apoio para a hipótese de que existe uma relação desse tipo, uma vez que o termo cúbico do grau de internacionalização, $\mathrm{GIO}^{3}$, não é significante. Adicionalmente, verifica-se que a inclusão do $\mathrm{GIO}^{3}$ não melhorou o modelo geral. $\mathrm{O} \mathrm{R}^{2}$ ajustado passou de 0,431 para 0,430 , bem como o Teste $\mathrm{F}$ foi reduzido de $(\mathrm{F}=12.477 ; P<0.001)$ para $(\mathrm{F}=11.623 ; P<0.001)$.

Em outras palavras, o poder explicativo do modelo não aumentou significativamente quando os termos quadrado e cúbico do grau de internacionalização $\left(\mathrm{GIO}^{2}\right.$ e $\left.\mathrm{GIO}^{3}\right)$ entraram na equação, de modo que o modelo linear se ajusta melhor aos dados do que o modelo não linear quadrático ou cúbico. Nesse sentido, rejeita-se a hipótese de que a relação entre o grau de internacionalização e desempenho em Empresas Multinacionais (EMNs) de economias em desenvolvimento é não linear, apresentando-se de forma quadrática (um ponto de inflexão) ou cúbica (dois pontos de inflexão).

Dessa forma, resumidamente, os resultados obtidos neste trabalho são: (a) a relação entre o grau de internacionalização e o desempenho dessas empresas é caracterizada por um comportamento linearnegativo, de modo que quanto maior o grau de internacionalização da empresa, menor tende a ser o seu desempenho; (b) o tamanho da empresa controla significativamente a relação entre o grau de internacionalização e o desempenho; e (c) a região de origem da empresa e alguns setores apresentam influência significante na análise dos três modelos.

Adicionalmente, foi realizada uma análise de regressão de dados em painel, uma vez que a utilização dessa técnica possibilita a obtenção de estimadores mais precisos e estatísticas de testes mais poderosas (Wooldridge, 2006). Através da realização do teste de Hausman, foi possível formalizar a melhor escolha entre os efeitos fixos e os aleatórios, conforme recomenda Hsiao (2003). Após a realização do teste de Hausman (qui-quadrado $=38,2517$; P-valor $=0,000139668$ ), constatouse que o P-valor do teste possui significância $(\mathrm{P}<0,05)$, demonstrando que a melhor escolha se dá pela utilização dos efeitos fixos (modelo 4) da Tabela 6. Desta forma, assume-se que existem efeitos específicos das EMNs da amostra e que tais efeitos são relacionados às variáveis independentes do modelo.

Com o intuito de responder à questão investigada neste artigo, os resultados obtidos foram analisados a partir da literatura em negócios internacionais, que aborda diversos aspectos que poderiam levar à perda de desempenho de uma empresa à medida que o seu grau de internacionalização aumenta. Para Ruigrok e Wagner (2003), com o aumento da expansão internacional, os custos de governança, bem como os custos de transação aumentam exponencialmente, devido à dispersão geográfica e cultural dos diretores e vários agentes da empresa multinacional. A teoria dos custos de transação sugere que o crescimento excessivo eventualmente aumentará os custos de governança e reduzirá os lucros (Jones \& Hill, 1988). Para Goerzen e Beamish (2003), "além desses encargos administrativos, a empresa pode incorrer na ineficiente alocação de 
recursos, devido ao aumento da dificuldade da empresa em alinhar as suas atividades de produção" (p. 1290).

Ainda, o estudo de Luo e Tung (2007) - sobre EMNs de economias em desenvolvimento apresenta possíveis razões para a relação linear negativa encontrada entre $o$ grau de internacionalização e o desempenho. Para esses autores, as EMNs de economias em desenvolvimento se internacionalizam sob uma perspectiva trampolim, teoria que descreve os prós e os contras da internacionalização no contexto das EMNs de países em desenvolvimento. Sob a perspectiva da teoria desenvolvida por esses autores (Luo \& Tung, 2007), acredita-se que a relação linear negativa encontrada neste trabalho deve-se ao fato de as EMNs de economias em desenvolvimento: (a) estarem na posição de Late Movers em mercados internacionais; (b) enfrentarem forte presença de concorrentes internacionais; (c) demonstrarem rápida mudança dos cenários tecnológicos e de mercado; (d) terem redução do ciclo de vida dos produtos e indústrias; e, sobretudo, (e) apresentarem deficiências em competências essenciais e ativos estratégicos.

Além disso, para Luo e Tung (2007), as EMNs de economias em desenvolvimento estão expostas a desafios exclusivos, tais como: (a) pobre governança corporativa; (b) dificuldades de integração e organização, após a internacionalização; (c) falta de experiência global, de competência administrativa e de experiência profissional; e (d) fraca inovação de produtos e processos. Neste sentido, acredita-se que todos esses fatores podem ter atuado para que a relação internacionalizaçãodesempenho tenha sido linear negativa para as EMNs de economias em desenvolvimento, apresentada neste estudo.

\section{Conclusão}

Com a emergência da internacionalização das empresas de economias em desenvolvimento, aumenta o interesse da comunidade científica pelo estudo com EMNs provenientes desses países. A abordagem dessas empresas como foco de estudo pode contribuir para a evolução da literatura na área de sua internacionalização, já que pode impulsionar mudanças nas teorias estabelecidas (CuervoCazurra, 2012; Ramamurti, 2012) ou até o desenvolvimento de novas teorias e modelos (Mathews, 2006).

Nesse sentido, a principal contribuição deste trabalho é a apresentação de uma análise empírica entre o grau de internacionalização e o desempenho das EMNs mais internacionalizadas de economias em desenvolvimento. Isso é relevante, já que os estudos existentes sob essa temática são majoritariamente realizados com EMNs de países desenvolvidos, e, aqueles que focaram economias em desenvolvimento, fizeram-no, geralmente, a partir de amostra com empresas provenientes de um único país ou região.

Baseado em estudos recentes sobre a relação internacionalização-desempenho com EMNs de economias em desenvolvimento (Chang, 2007; Chiao \& Yang, 2011; Contractor et al., 2007; H. Chen \& Hsu, 2010; Thomas, 2006), este trabalho hipotetizou que a relação entre o grau de internacionalização e o desempenho seria não linear quadrática ou cúbica para as 33 EMNs da amostra. Entretanto, os resultados obtidos com a análise de regressão múltipla não apoiaram essa hipótese, sendo encontrada uma relação significante linear-negativa entre o grau de internacionalização e o desempenho em EMNs de economias em desenvolvimento. Ou seja, os resultados sugerem que quanto maior o grau de internacionalização, menor tende a ser o desempenho dessas EMNs. Esse resultado é consistente com os resultados obtidos por Lin, Liu e Cheng (2011), que estudaram a relação internacionalização-desempenho em 179 empresas taiwanesas e por Wan (1998), em seu estudo com 120 EMNs de Hong Kong.

Uma importante implicação deste estudo para os gestores de negócios internacionais em economias em desenvolvimento é que, do ponto de vista dos custos de ser estrangeiro ou liability of 
foreignness (Hymer, 1960 como citado em Li, 2007; Zaheer, 1995), esse desempenho negativo pode estar relacionado à inexperiência das EMNs em mercados externos, falta de informação local, falta de familiaridade com a cultura estrangeira e, até mesmo, o tratamento discriminatório por parte do governo de outros países. Adicionalmente, a falta de experiência global, competência administrativa e experiência profissional são fatores que podem influenciar negativamente o desempenho das EMNs de economias em desenvolvimento em mercados estrangeiros (Luo \& Tung, 2007).

Outra importante implicação deste estudo para as decisões de negócios internacionais é que os benefícios da expansão internacional não surgem de uma hora pra outra, mas exigem paciência para serem alcançados. Compreendendo isso, os gerentes de EMNs de economias em desenvolvimento devem considerar que essas carências podem ser superadas pela própria expansão internacional, uma vez que essas EMNs iniciam sua internacionalização justamente para "compensar as suas fraquezas competitivas" (Luo \& Tung, 2007, p. 485). Seguindo essa lógica, a partir de certo ponto as EMNs "começam a ganhar conhecimento e experiência em mercados estrangeiros" (Thomas, 2006, p. 506). Tal constatação vai ao encontro do que propõe a teoria comportamental da internacionalização (Johanson \& Vahlne, 1977; Johanson \& Wiedersheim-Paul, 1975), segundo a qual "os obstáculos mais importantes para a internacionalização são a falta de conhecimento e recursos. Entretanto, esses obstáculos são reduzidos através de decisões incrementais e com a aprendizagem sobre os mercados e operações estrangeiras" (Johanson \& Wiedersheim-Paul, 1975, p. 306).

Desta forma, o resultado linear negativo não deve ser visto como um contraponto à teoria clássica de International Business, que enfatiza os benefícios do processo de internacionalização. $\mathrm{O}$ que entendemos por esse resultado é que a relação internacionalização-desempenho é uma análise relevante, mas que não deve ser considerada de maneira isolada quando se avaliam os resultados da internacionalização de uma empresa. Isso ocorre porque muitos outros fatores podem não apenas afetar a relação entre o grau de internacionalização e o desempenho dessas EMNs, mas também influenciar no desempenho de grandes empresas com atuação mundial.

\section{Limitações do estudo e sugestões para futuras pesquisas}

Este estudo possui limitações que devem ser superadas em futuras pesquisas. Primeiro, há uma forte concentração de empresas asiáticas na amostra, de modo que estudos futuros devem procurar obter maior equilíbrio entre os continentes. Embora tal característica da amostra tenha sido controlada através do país de origem (variável dummy), há implicações naturais no que diz respeito à questão da generalização dos resultados. Essa disparidade em favor das empresas asiáticas deve-se, sobretudo, à ascensão e, por conseguinte, maior quantidade de EMNs asiáticas no ranking elaborado pela UNCTAD, das 100 empresas mais internacionalizadas de economias em desenvolvimento, relatório utilizado para obtenção da amostra do presente estudo.

Em segundo lugar, apesar das análises estatísticas terem sido apoiadas por significância e estarem de acordo com os pressupostos da regressão múltipla, entende-se que seria oportuno aumentar o número de empresas estudadas. Isso seria possível através da utilização de uma base de dados mais robusta, como, por exemplo, a Compustat Data, que dispõe de informações de empresas de capital aberto em praticamente todas as bolsas de valores ao redor do mundo.

Finalmente, o modelo utilizado para avaliação da relação internacionalização-desempenho deve ampliar o escopo da estratégia de internacionalização. Estudos recentes parecem convergir no sentido de que a avaliação da relação entre o grau de internacionalização e o desempenho das EMNs deve considerar muitos outros aspectos inerentes a uma estratégia de internacionalização. Portanto, algumas linhas de investigação futura poderiam ser:

Avaliar se as EMNs que possuem executivos com mais experiência internacional e alto grau de governança corporativa são mais rentáveis do que seus concorrentes com profissionais menos experientes e fracos padrões de governança (Hennart, 2011; Herrmann \& Datta, 2005); 
Levar em consideração aspectos como o modo de entrada, objetivos da internacionalização e o ritmo da internacionalização (Wagner \& Ruigrok, 2004);

Estabelecer variáveis que possam eventualmente atuar como moderadoras da relação entre o grau de internacionalização e o desempenho, tais como: (a) velocidade da expansão internacional (Chang, 2007; Vermeulen \& Barkema, 2002); (b) escopo geográfico (Chang, 2007; Geringer et al., 1989); (c) gastos em publicidade (Delios \& Beamish, 1999; Lu \& Beamish, 2004; Siddharthan \& Lall, 1982); e (d) intensidade de P\&D (Lu \& Beamish, 2001; Pantzalis, 2001; Qian, 2002; Severn \& Laurence, 1974; Siddharthan \& Lall, 1982);

Analisar a relação internacionalização-desempenho com a variável dependente mensurada como os custos operacionais em relação ao faturamento, tendo em vista que Wagner e Ruigrok (2004), em sua revisão da literatura sobre internacionalização-desempenho, encontraram que medidas baseadas em custos resultaram em efeitos significativamente maiores do que ROA e ROS. Além disso, o Q de Tobin também parece ser uma alternativa interessante, pois consiste numa medida de desempenho baseada no mercado e não apenas contábil (Morck \& Yeung, 1991; Pantzalis, 2001); e

Identificar a relação de causalidade entre internacionalização-desempenho a partir de grupo de controle com empresas semelhantes, mas com operações puramente nacionais. Isso permite isolar a influência da variável internacionalização no desempenho.

\section{Referências}

Annavarjula, M., \& Beldona, S. (2000). Multinationality-performance relationship: a review and reconceptualization. International Journal of Organizational Analysis, 8(1), 48-67. doi: $10.1108 / \mathrm{eb} 028910$

Bobillo, A. M., López-Iturriaga, F., \& Tejerina-Gaite, F. (2010). Firm performance and international diversification: the internal and external competitive advantages. International Business Review, 19(6), 607-618. doi: 10.1016/j.ibusrev.2010.03.006

Brewer, H. L. (1981). Investor benefits from corporate international diversification. Journal of Financial and Quantitative Analysis, 16(1), 113-126. doi: 10.2307/2330669

Buckley, P. J., \& Casson, M. C. (2009). The internalization theory of the multinational enterprise: a review of the progress of a research agenda after 30 years. Journal of International Business Studies, 40(9), 1563-1580. doi: 10.1057/jibs.2009.49

Buhner, R. (1987). Assessing international diversification of West German corporations. Strategic Management Journal, 8(1), 25-37. doi: 10.1002/smj.4250080104

Capar, N., \& Kotabe, M. (2003). The relationship between international diversification and performance in service firms. Journal of International Business Studies, 34(4), 345-355. doi: 10.1057/palgrave.jibs. 8400036

Chang, J. (2007). International expansion path, speed, product diversification and performance among emerging-market MNEs: evidence from Asia-Pacific multinational companies. Asian Business and Management, 6(4), 331-353. doi: 10.1057/palgrave.abm.9200228

Chen, H., \& Hsu, C. W. (2010). Internationalization, resource allocation and firm performance. Industrial Marketing Management, 39(7), 1103-1110. doi: 10.1016/j.indmarman.2009.10.001

Chen, S., \& Tan, H. (2012). Region effects in the internationalization-performance relationship in Chinese firms. Journal of World Business, 47(1), 73-80. doi: 10.1016/j.jwb.2010.10.022 
Chiao, Y. C., \& Yang, K. P. (2011). Internationalization, intangible assets and Taiwanese SME's performance: evidence of an Asian newly-industrialized economy. African Journal of Business Management, 5(3), 641-655. doi: 10.5897/AJBM09.225

Collins, J. M. (1990). A market performance comparison of U.S firms active in domestic, developed and developing countries. Journal of International Business Studies, 21(2), 271-287. doi: 10.1057/palgrave.jibs.8490335

Contractor, F. J., Kumar, V., \& Kundu, S. K. (2007). Nature of the relationship between international expansion and performance: the case of emerging market firms. Journal of World Business, 42(4), 401-417. doi: 10.1016/j.jwb.2007.06.003

Contractor, F. J., Kundu, S. K., \& Hsu, C.-C. (2003). A three-stage theory of expansion of international expansion: the link between multinationality and performance in the service sector. Journal of International Business Studies, 34(1), 5-18. doi: 10.1057/palgrave.jibs.8400003

Cuervo-Cazurra, A. (2012). Extending theory by analyzing developing country multinational companies: solving the goldilocks debate. Global Strategy Journal, 2(3), 153-167. doi: 10.1111/j.2042-5805.2012.01039.x

Daniels, J. D., \& Bracker, J. (1989). Profit performance: do foreign operations make a difference? Management International Review, 29(1), 46-56.

Delios, A., \& Beamish, P. W. (1999). Geographic scope, product diversification and corporate performance of Japanese firms. Strategic Management Journal, 20(8), 711-727. doi: 10.1002/(SICI)1097-0266(199908)20:8<711::AID-SMJ41>3.0.CO;2-8

Dunning, J. H. (1988). The eclectic paradigm of international production: a restatement and some possible extensions. Journal of International Business Studies, 19(1), 1-31. doi: 10.1057/palgrave.jibs. 8490372

Dunning, J. H. (2000). The eclectic paradigm as an envelope for economic and business theories of MNE activity. International Business Review, 9(2), 163-190. doi: 10.1016/S09695931(99)00035-9

Gaur, S. A., \& Kumar, V. (2009). International diversification, business group affiliation and firm performance: empirical evidence from India. British Journal of Management, 20(2), 172-186. doi: 10.1111/j.1467-8551.2007.00558.x

Geringer, J. M., Beamish, P. W., \& Dacosta, R. C. (1989). Diversification strategy and internationalization: implications for MNE performance. Strategic Management Journal, 10(2), 109-119. doi: $10.1002 / \mathrm{smj} .4250100202$

Goerzen, A., \& Beamish, P. W. (2003). Geographic scope and multinational enterprise performance. Strategic Management Journal, 24(13), 1289-1306. doi: 10.1002/smj.357

Gomes, L., \& Ramaswamy, K. (1999). An empirical examination of the form of the relationship between multinationality and performance. Journal of International Business Studies, 30(1), 173-187. doi: 10.1057/palgrave.jibs. 8490065

Grant, R. M. (1987). Multinationality and performance among British manufacturing companies. Journal of International Business Studies, 18(3), 79-89. doi: 10.1057/palgrave.jibs.8490413

Grant, R. M., Jammine, A. P., \& Thomas, H. (1988). Diversity, diversification and profitability among british manufacturing companies. Academy Management Journal, 31(4), 771-801.

Haar, J. (1989). A comparative analysis of the profitability performance of the largest US, European and Japanese multinational enterprises. Management International Review, 29(3), 5-18. 
Habib, M. M., \& Victor, B. (1991). Strategy, structure, and performance of US manufacturing and service MNCs: a comparative analysis. Strategic Management Journal, 12(8), 589-606. doi: $10.1002 / \mathrm{smj} .4250120803$

Hair, J. F., Anderson, R. E., Tatham, R. L., \& Black, W. C. (1998). Multivariate data analysis. New Jersey: Upper Saddle River, Prentice Hall.

Han, K., Lee, S., \& Suk, D. (1998). Multinationality and firm performance. Multinational Business Review, 6(2), 63-70.

Hennart, J.-F. (2011). A theoretical assessment of the empirical literature on the impact of multinationality on performance. Global Strategy Journal, 1(1-2), 135-151. doi: 10.1002/gsj.8

Herrmann, P., \& Datta, D. K. (2005). Relationships between top management team characteristics and international diversification: an empirical investigation. British Journal of Management, 16(1), 69-78. doi: 10.1111/j.1467-8551.2005.00429.x

Hitt, M., Hoskisson, R., \& Kim, H. (1997). International diversification: effects on innovation and firm performance in product-diversified firms. Academy of Management Journal, 40(4), $767-$ 798. doi: $10.2307 / 256948$

Hoskisson, R. E. (1987). Multidivisional structure and performance: the contingency of diversification strategy. Academy of Management Journal, 30(4), 625-644. doi: 10.2307/256152

Hsiao, C. (2003). Analysis of panel data. Cambridge, United Kingdom: Cambridge University Press.

Hymer, S. (1960). The international operations of national firms: a study of foreign direct investment (Unpublished Dissertation). Massachusetts Institute of Technology, London, UK, England.

Johanson, J., \& Vahlne, J. E. (1977). The internationalization process of the firm: a model of market knowledge and increasing foreign market commitments. Journal of International Business Studies, 8(1), 23-32.

Johanson, J., \& Wiedersheim-Paul, F. (1975). The internationalization of the firm - four Swedish cases. Journal of Management Studies, 12(3), 305-323. doi: 10.1111/j.14676486.1975.tb00514.x

Jones, G. R., \& Hill, C. W. L. (1988). Transaction cost analysis of strategy-structure choice. Strategic Management Journal, 9(2), 159-172. doi: 10.1002/smj.4250090206

Kim, C. W., Hwang, P., \& Burgers, W. P. (1989). Global diversification strategy and corporate profit performance. Strategic Management Journal, 10(1), 45-57. doi: 10.1002/smj.4250100105

Kim, S. W., \& Lyn, E. O. (1987). Foreign direct investment theories, entry barriers, and reverse investments in U.S manufacturing industries. Journal of International Business Studies, 18(2), 53-66. doi: 10.1057/palgrave.jibs. 8490406

Kim, W. C., Hwang, P., \& Burgers, W. P. (1993). Multinationals' diversification and the risk-return tradeoff. Strategic Management Journal, 14(4), 275-286. doi: 10.1002/smj.4250140404

Kobrin, S. J. (1991). An empirical analysis of the determinants of global integration. Strategic Management Journal, 12(1), 17-31. doi: 10.1002/smj.4250120904

Kogut, B. (1985). Designing global strategies: profiting from operating flexibility. Sloan Management Review, 27(1), 27-38. 
Kotabe, M., Srinivasan, S. S., \& Aulakh, P. S. (2002). Multinationality and firm performance: the moderating role of R\&D and marketing capabilities. Journal of International Business Studies, 33(1), 79-97.

Li, L. (2005). Is regional strategy more effective than global strategy in the US service industries? Management International Review, 45(1), 37-57.

Li, L. (2007). Multinationality and performance: a synthetic review and research agenda. International Journal of Management Reviews, 9(2), 117-139. doi: 10.1111/j.1468-2370.2007.00205.x

Lin, W. T., Liu, Y., \& Cheng, K. Y. (2011). The internationalization and performance of a firm: moderating effect of a firm's behavior. Journal of international management, 17(1), 83-95. doi: 10.1016/j.intman.2010.12.004

Lu, J. W., \& Beamish, P. W. (2001). The internationalization and performance of SMEs. Strategic Management Journal, 22(6-7), 565-586. doi: 10.1002/smj.184

Lu, J. W., \& Beamish, P. W. (2004). International diversification and firm performance: the S-curve hypothesis. Academy of management journal, 47(4), 598-609. doi: 10.2307/20159604

Luo, Y., \& Tung, R. L. (2007). International expansion of emerging market enterprises: a springboard perspective. Journal of International Business Studies, 38(4), 481-498. doi: 10.1057/palgrave.jibs. 8400275

Mathews, J. A. (2006). Dragon multinationals: new players in $21^{\text {st }}$ century globalization. Asia Pacific Journal of Management, 23(1), 5-27. doi: 10.1007/s10490-006-6113-0

Michel, A., \& Shaked, I. (1986). Multinational corporations vs. domestic corporations: financial performance and characteristics. Journal of International Business Studies, 17(3), 89-100.

Morck, R., \& Yeung, B. (1991). Why investors value multinationality? Journal of Business, 64(2), 165-187. doi: $10.1086 / 296532$

Pantzalis, C. (2001). Does location matter? An empirical analysis of geographic scope and MNC market valuation. Journal of International Business Studies, 32(1), 133-155.

Qian, G. (1998). Determinants of profit performance for the largest US. firms 1981-92. Multinational Business Review, 6(2), 44-51.

Qian, G. (2002). Multinationality, product diversification, and profitability of US emerging and medium sized enterprises. Journal of Business Venturing, 17(6), 611-633. doi: 10.1016/S08839026(01)00080-5

Ramamurti, R. (2012). What is really different about emerging market multinationals? Global Strategy Journal, 2(1), 41-47. doi: 10.1002/gsj.1025

Riahi-Belkaoui, A. (1998). The effects of the degree of internationalization on firm performance. International Business Review, 7(3), 315-321. doi: 10.1016/S0969-5931(98)00013-4

Rugman, A. M. (1986). New theories of the multinational enterprise: an assessment of internalization theory. Bulletin of Economic Research, 38(2), 101-118. doi: 10.1111/j.14678586.1986.tb00208.x

Ruigrok, W., \& Wagner, H. (2003). Internationalization and performance: an organizational learning perspective. Management International Review, 43(1), 63-83.

Severn, A. K., \& Laurence, M. M. (1974). Direct investment, research intensity, and profitability. Journal of Financial and Quantitative Analysis, 9(2), 181-190. doi: 10.2307/2330094 
Siddharthan, N. S., \& Lall, S. (1982). Recent growth of the largest US. multinationals. Oxford Bulletin of Economics and Statistics, 44(1), 1-13. doi: 10.1111/j.1468-0084.1982.mp44001001.x

Sullivan, D. (1994). Measuring the degree of internationalization of a firm. Journal of International Business Studies, 25(2), 325-342. doi: 10.1057/palgrave.jibs.8490203

Sundaram, A. K., \& Black, J. S. (1992). The environment and internal organization of multinational enterprises. Academy of Management Review, 17(4), 729-757. doi: 10.5465/AMR.1992.4279065

Tallman, S., \& Li, J. (1996). Effects of international diversity and product diversity on the performance of multinational firms. Academy of Management Journal, 39(1), 179-196. doi: $10.2307 / 256635$

Thomas, D. E. (2006). International diversification and firm performance in Mexican firms: a curvilinear relationship? Journal of Business Research, 59(4), 501-507. doi: 10.1016/j.jbusres.2005.08.008

United Nations Conference on Trade and Development. (2004). The shift towards services (World Investment Report/2004), New York and Geneva, Division on Investment and Enterprise, United Nations.

United Nations Conference on Trade and Development. (2005). Transnational corporations and the internationalization of $R \& D$ (World Investment Report/2005), New York and Geneva, Division on Investment and Enterprise, United Nations.

United Nations Conference on Trade and Development. (2006). FDI from developing and transition economies: implications for development (World Investment Report/2006), New York and Geneva, Division on Investment and Enterprise, United Nations.

United Nations Conference on Trade and Development. (2007). Transnational corporations, extractive industries and development (World Investment Report/2007), New York and Geneva, Division on Investment and Enterprise, United Nations.

United Nations Conference on Trade and Development. (2008). Transnational corporations and the infrastructure challenge (World Investment Report/2008), New York and Geneva, Division on Investment and Enterprise, United Nations.

United Nations Conference on Trade and Development. (2009). Transnational corporations, agricultural production and development (World Investment Report/2009), New York and Geneva, Division on Investment and Enterprise, United Nations.

United Nations Conference on Trade and Development. (2011). Non-equity modes of international production and development (World Investment Report/2011), New York and Geneva, Division on Investment and Enterprise, United Nations.

Vermeulen, F., \& Barkema, H. (2002). Pace, rhythm, and scope: process dependence in building a profitable multinational corporation. Strategic Management Journal, 23(7), 637-653. doi: $10.1002 / \mathrm{smj} .243$

Wagner, H., \& Ruigrok, W. (2004). Internationalization and performance: a meta-analytic review and future research directions. Proceedings of the Academy of International Business annual meeting, Stockholm, Sweden.

Wan, C. C. (1998). International diversification, industrial diversification and firm performance of Hong Kong MNCs. Asian Pacific Journal of Management, 15(2), 205-217. doi: 10.1023/A:1015489514744 
Wernerfelt, B. (1984). A resource-based view of the firm. Strategic Management Journal, 5(2), 171180. doi: $10.1002 / \mathrm{smj} .4250050207$

Wooldridge, J. M. (2006). Introdução à economia: uma abordagem moderna. São Paulo: Thomson.

Zaheer, S. (1995). Overcoming the liability of foreignness. Academy of Management Journal, 38(2), 341-363. doi: $10.2307 / 256683$ 